\title{
Progress in nuclear data for accelerator applications in Europe
}

\author{
F. Goldenbaum ${ }^{\mathrm{a}}$ \\ Institut für Kernphysik, Forschungszentrum Jülich GmbH, 52428 Jülich, Germany
}

\begin{abstract}
This contribution essentially will be divided into two parts: First, a brief overview on topical accelerator applications in Europe, a selection of the European 6th framework accelerator and ADS programs will be given, second the emphasis will be put on selected nuclear data required for designing facilities planned or even under construction. In this second part the progress on nuclear data in the EU FP6 Integrated Project IP-EUROTRANS (specifically NUDATRA) is summarized. For proton-induced reactions in the energy range of $200-2500 \mathrm{MeV}$ experimental data and model comparisons are shown on total and double differential production cross sections of $\mathrm{H}$ - and $\mathrm{He}$-isotopes and intermediate mass fragments.
\end{abstract}

\section{Introduction}

The spectrum of nuclear data needed for accelerator applications is extensive, versatile and complex. The complexity of this vivid field is reflected in the large number of European (and world wide) projects currently supported in the 6th framework programs of the EU. First, few of them will be briefly presented in this contribution in order to give a coarse overview of ongoing accelerator application actions in Europe. To a large extend community-research infrastructure activities and integrated projects of FP6 EU-programs as e.g., CARE (Coordinated Accelerator Research for Europe) [1], FAIR (Facility for Antiproton and Heavy Ion Research) [2], EU-LIFE (Light Ion facility Europe) or IP EUROTRANS (European Research Programme for the Transmutation of high level nuclear waste in an accelerator driven system) [3] contain domains (e.g., NUDATRA-Nuclear Data for Transmutation) devoted to compiling and providing nuclear data indispensable for realization of large scale facilities.

In the framework of designing such facilities as for example subcritical assemblies, intense pulsed spallation neutron sources (SNS [8], JSNS [7], ESSI [6]), antiproton facilities (FAIR), accelerator-driven nuclear reactors, nuclear waste transmutation plants, energy amplifier systems, proton drivers for future neutrino factories (BENE-Beams for European Neutrino Experiments) and also for the application for radioactive beams, improved accuracy nuclear data are of great importance not only for shielding layouts, but also for estimation of damage in target and structural materials. Under irradiation the structural damages of materials used in construction of the facilities manifest themselves as atomic displacements, radiotoxicity, chemical corrosion and embrittlement. The expected radiation-induced damage of materials employed is mainly due to:

- helium gas production,

- elastic scattering of neutrons, charged particles, and in particular intermediate mass fragments and heavy residuals produced in spallation reactions.

Compared to the extensively studied radiation damage in fission reactors the damage in spallation neutron sources is

\footnotetext{
${ }^{a}$ Presenting author, e-mail: f.goldenbaum@fz-juelich.de
}

characterized by an about 500 times larger ratio of produced helium gas per atom to displacements per atom (He/dpa) in materials which are directly exposed to the incident proton beam. The second part of this contribution will focus on the latest progress on nuclear data achieved in the NUDATRA domain of the IP EUROTRANS. The objective of this project is to provide reliable and comprehensive experimental data serving as benchmarks for code development and validation in the $200-2000 \mathrm{MeV}$ energy range. To scrutinize several of such codes and to calculate as reliably as possible quantities related to high energy reactions, hadronic interaction lengths, reaction cross sections, average particle multiplicities, particle multiplicity and double differential energy distributions need to be investigated. In this context the latest results of crucial experiments performed at GSI and COSY essentially on helium and intermediate mass production will be presented and compared to model predictions.

\section{European accelerator driven projects}

The number of small and medium sized accelerator driven projects in Europe is quite large. New ideas and initiatives are emerging and certainly worth being mentioned, however let us here restrict the discussion to probably the most important forum consulted by the European Commission for decision taking on supporting future facilities in Europe called ESFRI (European Strategy Forum on Research Infrastructures) [4].

\subsection{ESFRI}

The ESFRI Roadmap identifies new Research Infrastructure (RI) of European interest corresponding to the long term needs of the European research communities, covering all scientific areas, regardless of possible location. Potential new RI (or major upgrade) identified are likely to be realized in the next 10 to 20 years. Therefore they may have different degrees of maturity. The ESFRI roadmap is an on-going process; therefore this roadmap will be periodically updated. The first revision of roadmap will already start early 2007. Following a request from the European Commission, ESFRI 
decided to compile a list of opportunities in order to assist the Commission in the preparation of its proposal for the Seventh Framework programme (FP7). In autumn 2006 ESFRI agreed on a first list of 35 mature proposals for new (or major upgrade of) facilities of European interest covering seven key research areas (environmental sciences; energy; materials sciences; astrophysics, astronomy, particle and nuclear physics; biomedical and life sciences; social science and the humanities; computation, data treatment). Those ones listed in the 2006 report [4] and related to accelerator (and reactor) applications are itemized in the following:

\section{Energy:}

- IFMIF int. fusion materials irradiation facility (10 MW high flux n-source),

- JHR high flux research reactor for fission reactors materials testing.

Material science:

- ELI extreme light intensity attosecond pulse laser,

- ESFR upgrade of the European synchrotron radiation facility (in 7 years),

- ESS-I Eur. spallation neutron source for n-spectroscopy,

- Eur. XFEL hard X-ray Free electron laser in Hamburg,

- ILL 20/20 upgrade of the European neutron spectroscopy facility (2 phases),

- IRUVX-FEL infrared to soft X-ray free electron lasers (in 5 user facilities).

Astro-, nuclear and particle physics:

- FAIR Facility for antiproton and ion research,

- SPIRAL2 production and study of rare isotope,

- LHC large hadron collider at CERN.

\subsection{Accelerator R\&D projects (EU)}

The European Commission provides a variety of instruments (I3 - Integrated Infrastructure Initiatives, DS - Design Studies, Network Activities, NEST - New and Emerging Science and Technology) to strengthen and financially support the high energy physics European community in order to play a leadership role in i) the improvement of existing accelerators ii) the development of new accelerators. To name a few such instruments there are, e.g.:

- CARE Coordinated Accelerator Research in Europe (I3),

- BENE Beams for European Neutrino Experiments (part of CARE, Network Activity),

- EUROTeV European Design study Towards a global TeV linear Collider (plays a major role for the ILC (int. linear collider), (Design Study),

- EURISOL European Isotope Separation Online Radioactive Ion Beam facility, (Design Study),

- EUROLEAP European laser electron controlled acceleration in plasmas to GeV energy range, (NEST).

The duration of such projects is $3-5$ years and generally about roughly $1 / 3$ of the total costs is covered by EU contributions.

\subsection{Multi MW-target projects}

Doubtless and in common for any high intensity accelerator driven MW target project nuclear data are indispensable for:

- performance optimization (choice of material, geometry, secondary particle production),

- life time assessment (aging, material damage as dpa, gas production, embrittlement in window and target, container, corrosion, composition modifications),

- radioprotection (activation, radioactive inventory, shortlived residue activity, shielding high-energy neutrons),

- waste management (long-lived residue radiotoxicity).

Very briefly some large scale facilities currently planned or under construction in Europe facing during their design these kind of issues will be adumbrated in the following:

\subsubsection{FAIR}

The FAIR (Facility for Antiproton and Ion Research) [2] has been presented as large scale international accelerator facility of the next generation capable of producing primary beams of protons (up to $30 \mathrm{GeV}, 2.5 \times 10^{13} / \mathrm{s}$ ), heavy ions (up to Uranium, up to $25 \mathrm{GeV} / \mathrm{u}, 10^{10} / \mathrm{s}$ ) and secondary beams of radioactive ions (up to $2 \mathrm{GeV} / \mathrm{u}$ ) and antiprotons (up to $30 \mathrm{GeV}$, up to $7 \times 10^{10} / \mathrm{h}$ ). For the antiproton production it is planned to bunch compress the $29 \mathrm{GeV}$ protons on the production target to $50 \mathrm{~ns}$. FAIR builds on the experience and technological developments already made at the existing GSI facility, and incorporates new technological concepts. At its heart is a double ring facility with a circumference of 1100 meters. A system of cooler-storage rings for effective beam cooling at high energies and various experimental halls will be connected to the facility. The new facility will be organized as a European/international research centre.

\subsubsection{Neutrino factories}

Intense neutrino factories as currently lively discussed in the EU CARE integrated infrastructure initiative [1] and the BENE network activity [5] employ very powerful MW proton drivers and produce finally neutrinos from $\pi$ and $\mu$ decay, respectively. These kind of facilities aim at $v$-production rates of up to $10^{21}$ useful $\mu$-decays/year.

An alternative scenario of producing intense $v$-beams is currently proposed by the decay of stored beta-active emitters instead of a $\mu$ :

$$
\begin{array}{ccc}
{ }_{2}^{6} \mathrm{He} \rightarrow \quad{ }_{3}^{6} \mathrm{Li}+e^{-}+\bar{v}_{e} & T_{1 / 2}=0.8 \mathrm{~s} \\
{ }_{10}^{18} \mathrm{Ne} \rightarrow \quad{ }_{9}^{18} \mathrm{Li}+e^{+}+v_{e} & T_{1 / 2}=1.7 \mathrm{~s} .
\end{array}
$$

As shown in figure 1 the He-production could be performed by converter technology using spallation neutrons from water cooled $\mathrm{W}$ or liquid $\mathrm{Pb}$ on $\mathrm{BeO}$ concentric cylinders via the reaction $\mathrm{n}+{ }^{9} \mathrm{Be} \rightarrow{ }^{6} \mathrm{He}+{ }^{4} \mathrm{He}$. A nominal production rate of $5 \times 10^{13}$ ions/s can be achieved. For the ${ }^{18} \mathrm{Ne}$-production a spallation of close-by target nuclides ${ }^{18} \mathrm{Ne}$ from $\mathrm{MgO}$ ${ }_{12}^{24} \mathrm{Mg}\left(\mathrm{p}, \mathrm{p}_{3} \mathrm{n}_{4}\right)_{10}^{18} \mathrm{Ne}$ is assumed to be a realistic reaction-the 


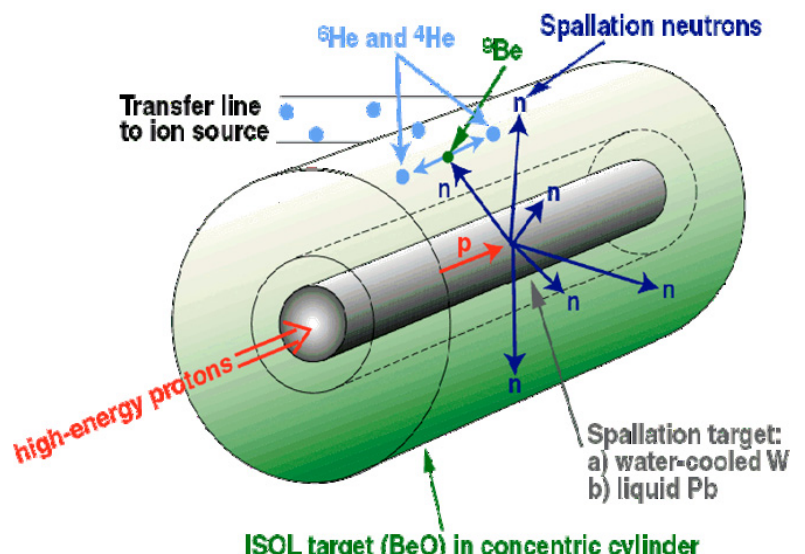

Fig. 1. ${ }^{6} \mathrm{He}$-production by converter technology using spallation neutrons.

beam hitting directly the oxide target. However for a few $\mathrm{GeV}$ proton beam and $200 \mathrm{~kW}$ dc power the estimated production rate will be more than one order of magnitude too low. Novel production scenarios will be required. For the production of the specific ions, usually an isotope separation (e.g., ISOL) is following the intense proton driver. These ions are accelerated to final energies and stored in large $(\mathrm{km})$ decay rings at high $\gamma\left({ }^{6} \mathrm{He}: \gamma \sim 150,{ }^{18} \mathrm{Ne}: \gamma \sim 60\right)$ for providing neutrino sources to experiments. Post accelerating the parent ions to relativistic $\gamma_{\max }$ has the advantage of boosted neutrino energy spectra and forward focussing of neutrinos: $\theta \leq 1 / \gamma$. Two (or more) different parent ions can be used for $v$ and $\bar{v}$-beams on $\beta^{+}$and $\beta^{-}$-decay. The physics applications of beta beams are primarily neutrino oscillations $v_{e} \leftrightarrow v_{\mu}$ (in particularly the single flavor decays as of eqs. (1) and (2)) and CP violation studies, but also measurements on cross sections of $v$-nucleus interactions. By far however not all technical issues are addressed and solved yet.

\subsubsection{Neutron spallation source}

As a successor of the original 2003 ESS feasibility study resulting in a comprehensive technical report [6], the European Spallation Source Initiative (ESS-I) was established as an association in which the users of neutron facilities, and regional consortia interested in hosting a new facility collaborate to prepare the case and the planning for a next generation neutron spallation source in Europe. The objectives of the ESS-I are to:

- gather all those interested in building at the earliest possible occasion a next generation top tier neutron source in Europe,

- stimulate, coordinate where necessary and possibly engage in all activities that need to be carried out before a decision to recommence baseline engineering, construction planning and detailed site assessment can be taken,

- act as the interlocutor to national governments and funding agencies, the EU and the ESF in matters regarding the initiative,

- have one European counterpart to SNS and the JSNS as part of J-PARC.

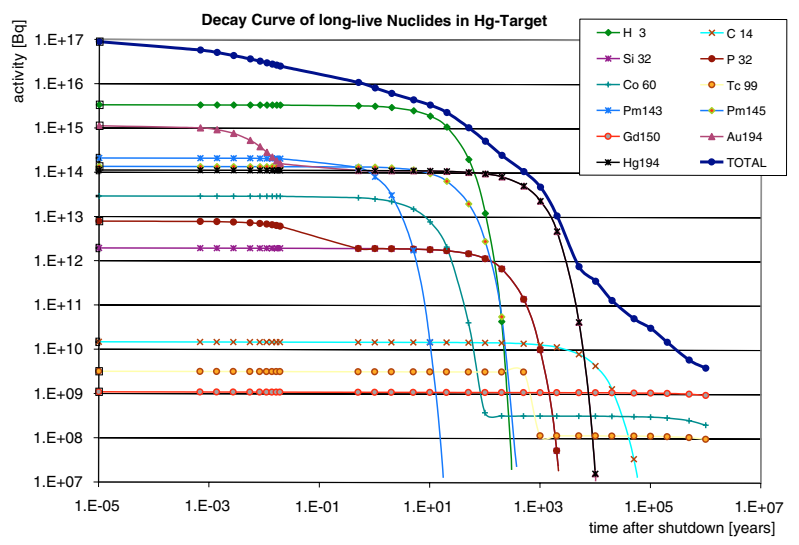

Fig. 2. Decay curves of long lived nuclides in Hg-target which determine the activity during operation and after 30 years of $5 \mathrm{MW}$ operation.

Related to the project laid down in the ESFRI roadmap, i.e., the $5 \mathrm{MW}$ long pulse target station, several countries/governments have declared to take an active part in the "preparatory phase" project of ESS within EU FP7 and shown interest in hosting the large scale facility. However appropriate conditions for international co-financing are not set up so far.

A baseline design phase resulted in a series of volumes [6] by the end of 2003 addressing the technical challenges that had been identified. As concerns the need for nuclear data, of crucial importance of any revised layout will be the optimization of the target/moderator/reflector assembly (geometry, material...) and the maximization of neutron flux. Carefully estimated also have to be the production rates in $\mathrm{GeV}$ p-induced spallation on heavy targets and the activities of target and structural materials during and after many years of full power operation. Figure 2 shows as an example for a $\mathrm{Hg}$ target the typical decay curves of individual nuclides during operation and after 30 years of $5 \mathrm{MW}$ operation as for ESS conditions. Of largest abundance is here the tritium activity up to $\mathrm{t}=10$ years as well as the ${ }^{194} \mathrm{Hg}$ and ${ }^{194} \mathrm{Au}$ activity dominating up to 1000 years and representing at the same time as long lived isotopes the nuclides of largest concern.

\section{Nuclear data EU-projects}

Already in the fifth framework program, projects aiming at new nuclear data measurements and evaluations as, e.g., HINDAS (High and Intermediate energy Nuclear Data for Accelerator-driven Systems) were supported by the EU. Within the three years program HINDAS provided a wealth of new nuclear reaction cross sections in the energy range between $20 \mathrm{MeV}$ and $2 \mathrm{GeV}$ on three elements of crutial importance for ADS systems: $\mathrm{Pb}$ as a target element, $\mathrm{U}$ as an actinide and $\mathrm{Fe}$ as a shielding element. As a natural extension or successor of the FP5 HINDAS project, in FP6, the project EUROTRANS (EUROpean Research Programme for the TRANSmutation of high level nuclear waste in an accelerator driven system) has been launched. Due to the lack of progress in implementing the radioactive waste disposal in geological repositories, the Partitioning and Transmutation (P\&T) 
strategy has been put forward. requirements. In addition, P\&T both will contribute to the sustainability of nuclear energy in those countries that pursue this source of energy, and will assist in combating global warming. In the Euratom Sixth Framework Program (FP6), one specific targeted research project on the study of the impact of P\&T (RED-IMPACT) and two integrated projects: (i) EUROPART (partitioning) and (ii) EUROTRANS (transmutation) have been launched. EUROTRANS benefits not only from the technological developments and scientific progress achieved in Europe during FP5, but also from worldwide co-operation (OECD/NEA, IAEA, USA, Japan, ISTC, etc.) in the field of P\&T. The FP5 $R \& D$ results in the areas of preliminary design studies of an experimental ADS, low-power coupling of an accelerator to the Masurca zero power reactor made sub-critical for the purpose of the MUSE programme, studies on transmutation fuels, heavy liquid metal technology and high-energy nuclear data are available as a basis for the advancement of this Integrated Project (IP). The EUROTRANS project is structured into one management and five technical Domains (DM) that are further subdivided into work packages and tasks.

\section{Selected nuclear data (NUDATRA)}

In the following the emphasis will be on EUROTRANS DM5 NUDATRA (Nuclear data for transmutation of nuclear waste). The goal of this domain is to improve nuclear data evaluated files and models which involves sensitivity analysis and validation of simulation tools, low and intermediate energy nuclear data measurements, nuclear data libraries evaluation at low and medium energies, and high energy experiments and modeling. In the following the focus is on NUDATRA WP5.4 - High energy experiments and modeling. This workpackage aims at the investigation of:

- pA (spallation) reactions in the GeV regime,

- data measured from exclusive experiments for testing, validating and developing theoretical models,

- double differential cross sections (DDXS) $d \sigma / d E d \Omega$ of light charged particles ( $\mathrm{LCP}=\mathrm{p}, \mathrm{d}, \mathrm{t},{ }^{3} \mathrm{He},{ }^{4} \mathrm{He} \ldots$ ) and intermediate mass fragments (IMFs, $Z \leq 16$ ) in spallation and fragmentation p-induced reactions $(0.1-2.5 \mathrm{GeV}, \mathrm{C}$ to $\mathrm{Au}$ ),

- reaction mechanism of $\mathrm{pN}$ reactions in terms of time scales, simultaneous or sequential emission of IMFs, origin of pre-equilibrium and evaporation processes.

\subsection{Light charged particle and IMF production}

As an example for light charged particle production, double differential energy spectra of ${ }^{1,2,3} \mathrm{H}$ and ${ }^{3,4} \mathrm{He}$ ejectiles following $1.2 \mathrm{GeV}$ p-induced reactions on Ta target as measured by NESSI collaboration at COSY-Jülich is shown for different angles in respect to the incident proton in figure 3 . The experimental data clearly feature two components, an evaporation component dominant for all angles and at low kinetic energies and a high energy component all the more pronounced the smaller the angle of the ejectile in respect to the incident proton is. Here [9] for the theoretical description the INCL2.0 [10]

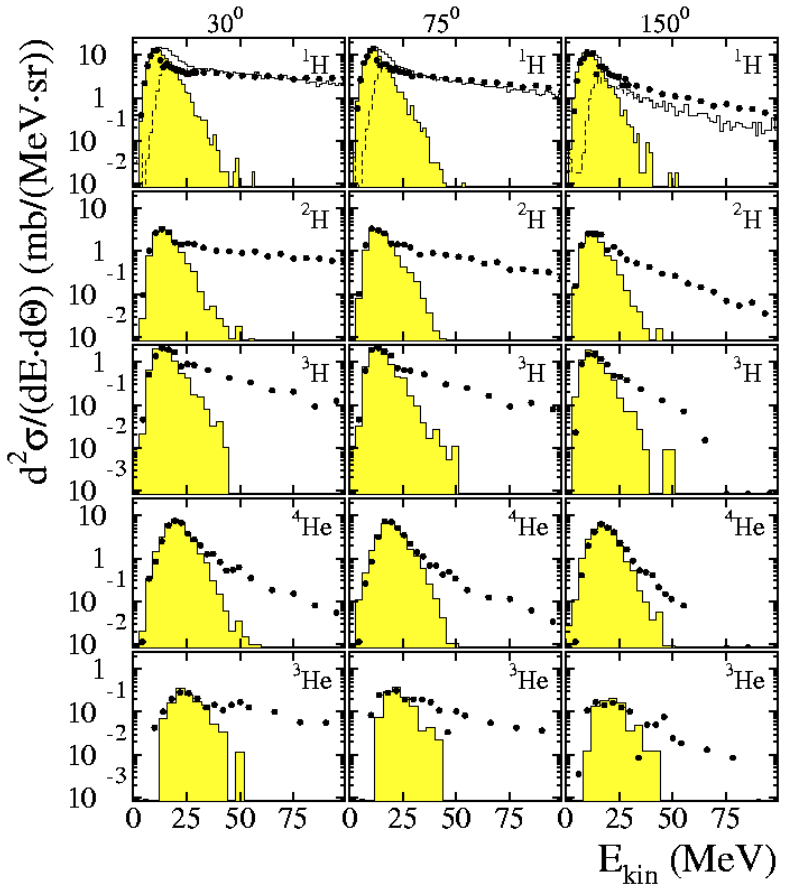

Fig. 3. Energy spectra of ${ }^{1,2,3} \mathrm{H}$ and ${ }^{3,4} \mathrm{He}$ for $1.2 \mathrm{GeV}$ p+Ta. dots: exp. data, shaded hist.: calculated evap. spectra, dashed hist.: preequilibrium protons as calculated by INCL2.0 [9].

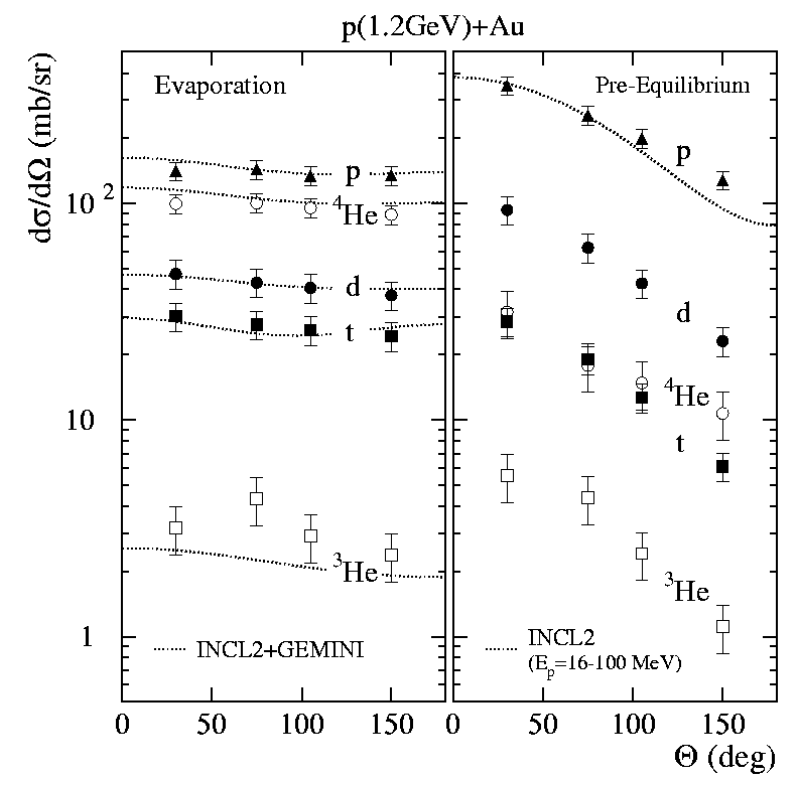

Fig. 4. Angular distributions of ${ }^{1,2,3} \mathrm{H}$ and ${ }^{3,4} \mathrm{He}$ for $1.2 \mathrm{GeV} \mathrm{p}+\mathrm{Au}$. symbols: exp. data, lines calculation by INCL2.0+GEMINI [9].

intranuclear cascade code is coupled to the evaporation code GEMINI [11]. Only for protons both components can be well described. Due to the lack of composite particle emission in the early phase of the reaction in the INCL2.0 model, the high energy tails of the spectra for $\mathrm{d}, \mathrm{t},{ }^{3,4} \mathrm{He}$ are not described by the calculations. The shape of the calculated evaporation component (shaded yellow histogram in fig. 3) however is well reflected also for composite particles. For $1.2 \mathrm{GeV}$ $\mathrm{p}+\mathrm{Au}$ in figure 4 , the angular distribution of disentangled 


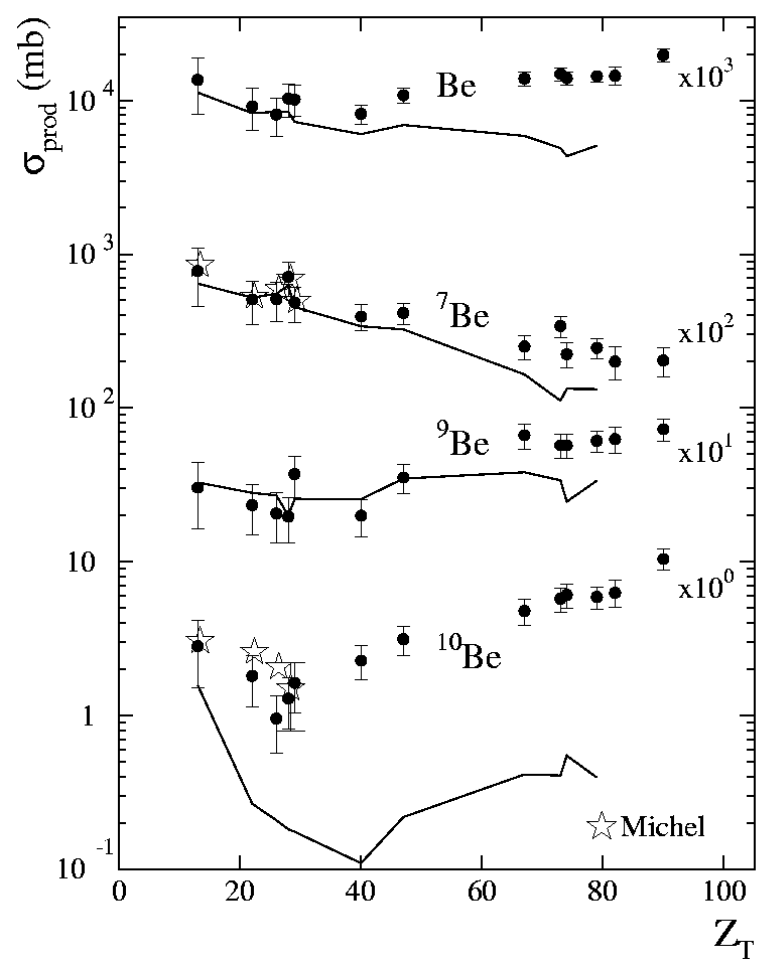

Fig. 5. Production cross section of ${ }^{7,9,10} \mathrm{Be}$ isotopes for $1.2 \mathrm{GeV} p+\mathrm{X}$. Black points: NESSI [9], empty stars: R. Michel [13] data, lines: calculation by INCL2.0+GEMINI.

evaporation (left panel) and pre-equilibrium (right panel) components are shown. For all particle species the evaporation exhibits an isotopic behaviour, while more directly emitted particles show larger forward/backward asymmetry. Note that for pre-equilibrium protons the angular dependence is well described in the INCL2.0 model. It would be certainly worth to compare the current experimental data [9] with the latest version of INCL4.3 [12] including a coalescence formalism allowing for the cluster emission of composite nucleons $\left(\mathrm{d}, \mathrm{t},{ }^{3,4} \mathrm{He}\right)$ in the early phase of the reaction.

The production cross sections of ${ }^{7,9,10} \mathrm{Be}$ isotopes for 1.2 $\mathrm{GeV}$ protons on different targets ( $\mathrm{C}$ to $\mathrm{Au})$ as well as the total $\sigma_{\mathrm{Be}}$ are shown in figure 5 . The production of all individual isotopes does not strongly depend on $Z$, respectively. When looking more carefully at the energy spectra of IMFs (not shown in this contribution), ones more as expected the combination of INCL2.0 + GEMINI fails to describe the high energy tails of the energy spectra. Nevertheless in figure 5 the calculated angle and energy integrated production cross sections agree generally rather well with the NESSI $[9,13]$ data, because the pre-equilibrium component amounts to the total cross section only on the percent level. The lines representing the model prediction are reflecting the ejectiles coming from evaporation model only, i.e., GEMINI. The experimental data on ${ }^{7} \mathrm{Be}$ and ${ }^{10} \mathrm{Be}$ ejectiles measured for low $Z$-targets by mass spectrometry [13] coincide with the systematics of the NESSI experiment [9]. In a similar presentation one would observe the multiplicity/production cross sections of the neutron rich $\mathrm{He}$ isotope $\left({ }^{6} \mathrm{He}\right)$ strongly increasing with atomic number $Z$ of the bombarded target (not explicitly shown here) - a

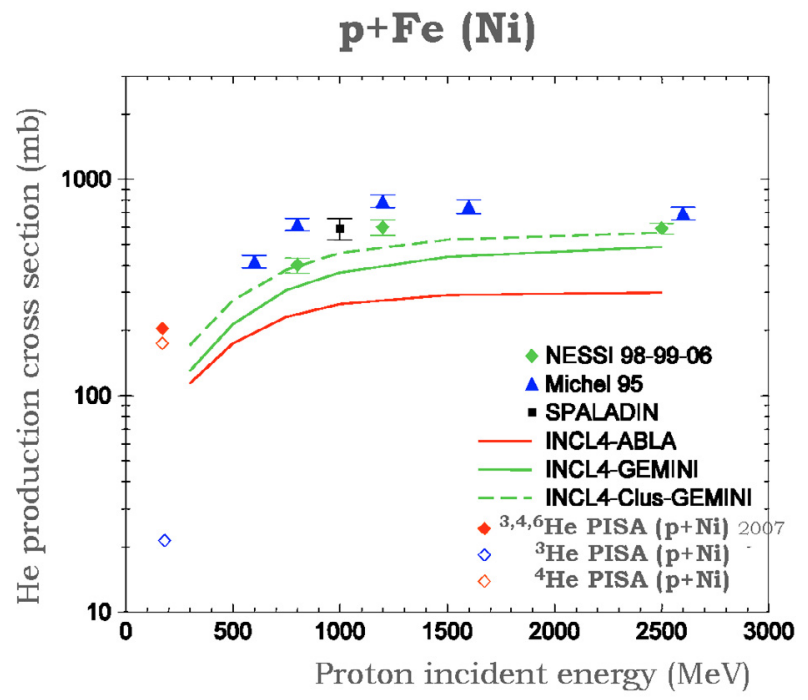

Fig. 6. Production cross section of ${ }^{3,4,6} \mathrm{He}$ isotopes as a function of incident proton beam energy. Symbols: NESSI, Hannover, SPALADIN, PISA data, curves: calculation by INCL4.3+GEMINI/ABLA.

very similar behavior as the one which is observed for the "neutron rich" triton. In contrast to the ${ }^{3,4} \mathrm{He}$ isotopes, for ${ }^{6} \mathrm{He}$ the INCL2.0+GEMINI calculations overestimates the experimental results of Herbach et al. [9] by approx. 30\%.

The international collaboration PISA (Proton Induced SpAllation) [14-17] is aiming at a quite similar physics program as NESSI, however with a completely different setup and at an internal beam location. At the internal beam of COSY the investigation of the reactions induced by protons on thin targets $\left(50-200 \mu \mathrm{g} / \mathrm{cm}^{2}\right)$ enables us to get the cross sections without the absorption and energy loss involved with the propagation of reaction products in the material of the target. The multiple circulation of the beam in the COSY ring is used to compensate for the small reaction rate of beamprotons with the thin targets. The advantage being higher statistics and more precise information on the very tails of double differential energy spectra. Isotope separation was done by combining the information from multi-channel-plates (time-of-flight), silicon detector telescopes and Bragg curve spectroscopy (energy deposited inside Bragg curve detectors) allowing for the separation of following isotopes: ${ }^{6} \mathrm{Li},{ }^{7} \mathrm{Li}$, ${ }^{8} \mathrm{Li}-{ }^{7} \mathrm{Be},{ }^{9} \mathrm{Be},{ }^{10} \mathrm{Be}-{ }^{10} \mathrm{~B},{ }^{11} \mathrm{~B}-{ }^{11} \mathrm{C},{ }^{12} \mathrm{C},{ }^{13} \mathrm{C},{ }^{14} \mathrm{C}$ and ${ }^{13} \mathrm{~N}$, ${ }^{14} \mathrm{~N}$ [17]. Measurements of these cross sections are important for providing benchmark data in the $\mathrm{GeV}$ incident p-energy range, understanding the complex reaction mechanism itself and testing the reliability of physical models describing the fast intranuclear cascade (INC) phase as well as the subsequent statistical decay from an equilibrated or thermalized hot nucleus. As already mentioned, a particular focus is on developing new models for the description of highly energetic composite particles [12].

As a function of incident proton beam energy the Heproduction cross sections on Fe measured by NESSI [9], Hannover [13], SPALADIN [18], and PISA [19] are compiled in figure 6. The latest data points of SPALADIN at $1 \mathrm{GeV}$ and PISA at $175 \mathrm{MeV}$ are also included. The SPALADIN result obtained in inverse kinematics of Fe on $\mathrm{p}$ at GSI shows a value 
slightly above the NESSI data, but is definitely still smaller than the systematics of R. Michel et al. [13]. The data shown here for PISA are for Ni reaction, but a comparison should be legitim, because $\mathrm{Fe}$ and $\mathrm{Ni}$ are very close in terms of atomic number. Note, that for the PISA data [19] the cross sections for the individual ${ }^{3,4,6} \mathrm{He}$ isotopes are given at $175 \mathrm{MeV}$. The Monte Carlo calculation getting closest to the available experimental $\mathrm{He}$ data is the INCL4-Clus-GEMINI version (dashed line in fig. 6), which accounts-using a coalescence approach-for cluster (here composite He particles) emitted in the first fast phase of the reaction. The two solid lines in figure 6 take into account only the He particles being emitted during the slow evaporation phase and therefore as expected the abundance of production cross sections is underestimated in INCL4 + ABLA or INCL4 + GEMNI, respectively.

Of great value and particular interest are the measurements performed by mass spectrometry $[13,20]$. The authors provide excitation functions in the whole energy range of interest, however in particular for light targets typically the measured He production cross sections do not coincide. The discrepancies between the two experimental methods for light targets is not yet understood. The huge amount of data collected for proton induced reactions will be valuable for the identification of deficiencies of existing INC/evaporation codes.

\subsection{Neutron production}

Of significant interest for a wide range of applications and fundamental research, in particular at the crux of spallation neutron sources, transmutation of nuclear waste in accelerator driven systems [21], and shielding issues are also neutron production double differential cross sections in $\mathrm{GeV}$ proton-induced spallation reactions. Although generally best described by INC + evaporation codes, neutrons are more difficult to detect than protons or LCP. Experimental double differential neutron production spectra represent a valuable observable also for validating new model developments or improvements $[12,22,24,25]$. It is also interesting to look at neutron multiplicities $M_{n}$, global properties of neutron spectra which are not easily revealed by their inspection. An extensive overview on the observable $M_{n}$ for both thin and thick (ejectiles induce secondary reactions) targets is compiled in refs. [25-28].

\section{Conclusion}

It has been shown, that a series of large scale accelerator facilities are currently planned or even under construction in Europe. The EU financially supports activities and provides within framework programs several instruments Some selected aspects within the Integrated Project EUROTRANS domain NUDATRA have been presented. Few experiments have been consulted to validate models with regard to reaction cross sections or reaction probabilities, charged particle production cross sections and angular- and energy-distributions for $\mathrm{GeV}$ proton induced reactions on various thin targets. PISA experiment, e.g., has shown to be able to measure the products of proton-nucleus collisions with Z-identification up to at least $\mathrm{Z}=16$ and isotope identification to masses up to $13-14$. A comprehensive comparison not only of light charged particles, but also of IMF particles with model predictions is in progress. Therefore the high energy experiments presented here provide an important set of benchmark data for the development and test of reliable new models capable of describing the emission of the high energy component of composite particles.

The author acknowledges gratefully the support of the European Community-Research Infrastructure Activity under FP6 "Structuring the European Research Area" programme (CARE-BENE, contract number RII3-CT-2003-506395 and HadronPhysics, contract number RII3-CT-2004-506078). The NESSI/PISA collaboration appreciates the financial support of the European Commission through the FP6 IP-EUROTRANS FI6W-CT-2004-516520.

\section{References}

1. http://care.lal.in2p3.fr/

2. http://www.gsi.de/fair/

3. http://nuklear-server.ka.fzk.de/eurotrans/

4. http://cordis.europa.eu/esfri/roadmap.htm (2006).

5. http://bene.web.cern.ch/bene/

6. ESS Project, Vol. 3, Tech. Rep., ISBN 3-89336-303-3 (2002).

7. S. Nagamiya, 9 th Int. Conf. on radiation shielding, Oct. 17-22nd, Tsukuba, Japan (1999).

8. Spallation Neutron Source SNS, http://www.ornl.gov/sns/

9. C.-M. Herbach et al., Nucl. Phys. A 765, 426 (2006).

10. J. Cugnon et al., Nucl. Phys. A 620, 475 (1997).

11. R.J. Charity et al., Nucl. Phys. A 483, 371 (1988).

12. A. Boudard et al., Nucl. Phys. A 740, 195 (2004).

13. R. Michel et al., Nucl. Instrum. Meth. B 103, 183 (1995).

14. http://www.fz-juelich.de/ikp/pisa

15. F. Goldenbaum et al., Nucl. Instrum. Meth. A 562, 733 (2005).

16. R. Barna et al., Nucl. Instrum. Meth. A 519, 610 (2003).

17. A. Bubak, Ph.D. thesis, Jül-4138, ISBN 0944-2952 (2004).

18. E. Le Gentil, Ph.D. thesis, Évry University, France (2006).

19. B. Piskor-Ignatowicz (2007) (private communication).

20. I. Leya et al., Nucl. Instrum. Meth. Phys. Res. B 229, 1 (2005).

21. H. Nifenecker et al., Nucl. Instrum. Meth. A 463, 428 (2001).

22. J. Cugnon et al., Nucl. Phys. A 625, 729 (1997).

23. S. Leray et al., Phys. Rev. C 65, 044621 (2002).

24. H. Duarte, Phys. Rev. C 75, 024611 (2007).

25. A. Boudard et al., Phys. Rev. C 66, 044615 (2002).

26. D. Filges et al., Eur. Phys. J. A 11, 467 (2001).

27. A. Letourneau et al., Nucl. Instrum. Meth. B 170, 299 (2000).

28. D. Hilscher et al., Nucl. Instrum. Meth. A 414, 100 (1998). 